Turk. J. Math. Comput. Sci.

12(2)(2020) 157-160

(C) MatDer

DOI : $10.47000 /$ tjmcs. 822830

\title{
On The Solution of an Infinite System of Discrete Equations
}

\author{
Anar AdiloĞLu Nabiev ${ }^{1, *}$ (iD, Mehmet Gürdal ${ }^{2}$ \\ ${ }^{1}$ Department of Computer Engineering, Süleyman Demirel University, 32260 Isparta, Turkey. \\ ${ }^{2}$ Department of Mathematics, Süleyman Demirel University, 32260 Isparta, Turkey.
}

Received: 07-11-2020 • Accepted: 13-12-2020

AвSTRACT. In this work, we construct the transformation operator for the infinite system of the difference equations

$$
a_{n-2} y_{n-2}+b_{n-1} y_{n-1}+c_{n} y_{n}+b_{n} y_{n+1}+a_{n} y_{n+2}=\lambda y_{n}(n=1,2, \ldots)
$$

where $a_{n} \neq 0, b_{n}, c_{n}(n=1,2,3, \ldots)$ are given complex numbers, investigate some important properties of the special solutions of the difference system.

2010 AMS Classification: 47B36, 47B39, 39A70

Keywords: Difference equation, Jacobi matrix, Transformation operators, Direct problem, Inverse problem.

\section{INTRODUCTION}

Consider the linear difference equation of the second order

$$
a_{n-1} y_{n-1}+b_{n} y_{n}+a_{n} y_{n+1}=\lambda y_{n}, n=1,2, \ldots
$$

where $\left\{y_{n}\right\}_{n=0}^{\infty}$ is an unknown infinite vector called a solution, $\lambda$ is a complex parameter and $a_{n} \neq 0, b_{n}$ are given complex numbers. G.Sh. Guseinov [1] investigated this infinite system of difference equations to define the three diagonal Jacobi matrix by the scattering data. He proved that if $a_{n}>0, \operatorname{Im} b_{n}=0(n=1,2, \ldots)$ and

$$
\sum_{n=1}^{\infty} n\left(\left|1-a_{n}\right|+\left|b_{n}\right|+\left|c_{n}\right|\right)<\infty
$$

then the scattering data for the equation (1.1) uniquely defined the sequences $\left(a_{n}\right)$ and $\left(b_{n}\right)$. In this work G.Sh. Guseinov improved the Marchenko method for the difference system (1.1) by constracting an analogue of the transformation operator for the Sturm-Liouville operator. Direct and inverse spectral problems for nonselfadjoint Jacobi matrices also were investigated by G.Sh. Guseinov [3] who gave a procedure for construction of a spectral function for the system (1.1). The full line inverse scattering problem for the second order difference system (1.1) in various statements were investigated in $[2,4,5]$ by using transformation operators and with application of the Marchenko method.

Nonselfadjoint difference operators of an even order was considered by Yu.L. Kishakevich [6] for constructing the Parsevall-Marchenko identity. Using matrix moment problem he showed how to obtain the Parsevall identity for the selfadjoint case. Recently, S. M Zagorodnyuk in the series of his studies [8,9] has considered the direct and inverse spectral problems for $(2 N+1)$ - diagonal Jacobi matrices. In these works the author used a different method for the construction of the spectral function and extended Guseinov's results to the case of $(2 N+1)$-diagonal complex

*Corresponding Author

Email addresses: anaradiloglu@sdu.edu.tr (A. Adiloğlu Nabiev), gurdalmehmet@ sdu.edu.tr (M. Gürdal) 
symmetric matrices. But in these studies the special Fourier type series representations of the solutions of an even order difference system was not considered and logically the direct and inverse scattering problems for the $(2 N+1)$ diagonal Jacobi matrices as generalization of G.Sh. Guseinov's result have not yet investigated.

In this work, as the first step of the investigation the direct and inverse scattering problems for the five-diagonal Jacobi matrices by the Marchenko method, we construct the transformation operator for the infinite system of the difference equations

$$
a_{n-2} y_{n-2}+b_{n-1} y_{n-1}+c_{n} y_{n}+b_{n} y_{n+1}+a_{n} y_{n+2}=\lambda y_{n}, n=1,2, \ldots
$$

where $a_{n} \neq 0, b_{n}, c_{n}(n=1,2,3, \ldots)$ are given complex numbers, and investigate some important properties of the special solutions of the system (1.2).

\section{The Transformation Operator for the Five Diagonal Jacobi Matrix}

We consider the infinite system of discrete equations

$$
\begin{gathered}
c_{1} y_{1}+b_{1} y_{2}+a_{1} y_{3}=\lambda y_{1}, \\
b_{1} y_{1}+c_{2} y_{2}+b_{2} y_{3}+a_{2} y_{4}=\lambda y_{2}, \\
a_{n-2} y_{n-2}+b_{n-1} y_{n-1}+c_{n} y_{n}+b_{n} y_{n+1}+a_{n} y_{n+2}=\lambda y_{n}, n=3,4, \ldots
\end{gathered}
$$

where $\left\{y_{n}\right\}_{n=1}^{\infty}$ is an unknown infinite vector called a solution, $\lambda$ is a complex parameter and $a_{n} \neq 0, b_{n}, c_{n}(n=$ $1,2,3, \ldots)$ are given complex numbers such that the series

$$
\sum_{n=1}^{\infty} n\left(\left|1-a_{n}\right|+\left|b_{n}\right|+\left|c_{n}\right|\right)
$$

converges. The equation (2.1) is equivalent to defining a vector $\left\{y_{n}\right\}_{n=-1}^{\infty}$ which satisfies

$$
a_{n-2} y_{n-2}+b_{n-1} y_{n-1}+c_{n} y_{n}+b_{n} y_{n+1}+a_{n} y_{n+2}=\lambda y_{n}, n=1,2, \ldots ; a_{-1}=a_{0}=1, b_{0}=0
$$

under conditions

$$
y_{-1}=0, y_{0}=0 .
$$

This system of difference equations can be written by the help of the five-diagonal Jacobi matrix in the form

$$
J Y=\lambda Y
$$

where $Y=\left(y_{1}, y_{2}, \ldots\right)^{T}$ and

$$
J=\left(\begin{array}{llllllll}
c_{1} & b_{1} & a_{1} & 0 & 0 & 0 & \ldots & \ldots \\
b_{1} & c_{2} & b_{2} & a_{2} & 0 & 0 & \ldots & \ldots \\
a_{1} & b_{2} & c_{3} & b_{3} & a_{3} & 0 & \ldots & \ldots \\
0 & a_{2} & b_{3} & c_{4} & b_{4} & a_{4} & 0 & . . \\
\ldots & \ldots & \ldots & \ldots & \ldots & \ldots & \ldots & \ldots
\end{array}\right) .
$$

Difference or discrete equations of type (2.3) often arises in various problems related to physics and engineering. The second order difference equation

$$
a_{n-1} y_{n-1}+b_{n} y_{n}+a_{n} y_{n+1}=\lambda y_{n}, n=1,2, \ldots
$$

is a simple discretization of the Sturm-Liouville equation

$$
-y^{\prime \prime}+q(x) y=\lambda y
$$

which has a lot of applications in physics and quantum mechanics [7].

If $a_{n}=1, b_{n}=0, c_{n}=0$ then for $\lambda=2 \cos 2 \xi$ Eq. (2.3) has the simple solution $f_{n}^{0}(\xi)=e^{i n \xi}$. Using this fact we require that the problem (2.3)-(2.4) has a solution $f_{n}(\xi)$ which is presented as

$$
f_{n}(\xi)=\alpha_{n} e^{i n \xi}\left(1+\sum_{m=1}^{\infty} K_{n, m} e^{i m \xi}\right), n=1,2, \ldots
$$

Here $\alpha_{n}$ and $K_{n, m}(n=1,2, \ldots ; m=1,2, \ldots)$ are complex numbers and satisfy the conditions

$$
\lim _{n \rightarrow \infty} \alpha_{n}=1, \lim _{n \rightarrow \infty} K_{n, m}=0(m=1,2, \ldots) .
$$

We can extend $K_{n, m}$ as $K_{n, m}=0$ for $m \leq 0$ if we need it elsewhere. 
The solution (2.5) of the discrete system (2.3) is called transformation operator for the fourth order difference operator and it is important in the investigation of the spectral problems, instantly, inverse scattering problems for the equation (2.3) (see $[1,3,7])$. Substituting $y_{n}=f_{n}(\xi)$ in equations (2.3) after some simple transformation we have

$$
\begin{gathered}
\alpha_{n}=\alpha_{n-2} a_{n-2}, \\
\alpha_{n-1} b_{n-1}+\alpha_{n} K_{n-2,1}-\alpha_{n} K_{n, 1}=0, \\
c_{n} \alpha_{n}+\alpha_{n} K_{n-2,2}-\alpha_{n} K_{n, 2}+\alpha_{n-1} b_{n-1} K_{n-1,1}=0, \\
b_{n} \alpha_{n+1}+\alpha_{n} K_{n-2,3}-\alpha_{n} K_{n, 3}+\alpha_{n-1} b_{n-1} K_{n-1,2}+\alpha_{n} c_{n} K_{n, 1}=0, \\
\alpha_{n} a_{n}^{2}-\alpha_{n}+\alpha_{n} K_{n-2,4}-\alpha_{n} K_{n, 4}+\alpha_{n-1} b_{n-1} K_{n-1,3}+ \\
+c_{n} \alpha_{n} K_{n, 2}+\alpha_{n+1} b_{n} K_{n+1,1}=0 \\
\alpha_{n} a_{n}^{2} K_{n+2, m}-\alpha_{n} K_{n, m}+\alpha_{n+1} b_{n} K_{n+1, m+1}+c_{n} \alpha_{n} K_{n, m+2}+ \\
+\alpha_{n-1} b_{n-1} K_{n-1, m+3}+\alpha_{n} K_{n-2, m+4}-\alpha_{n} K_{n, m+4}=0, m \geq 1 .
\end{gathered}
$$

Now from the equations (2.6)-(2.11) we can find that all these equations are equivalent to the following equations:

$$
\begin{gathered}
a_{n}^{2} K_{n+2, m}-K_{n, m}+\frac{\alpha_{n+1}}{\alpha_{n}} b_{n} K_{n+1, m+1}+c_{n} K_{n, m+2}+ \\
+\frac{\alpha_{n-1}}{\alpha_{n}} b_{n-1} K_{n-1, m+3}+K_{n-2, m+4}-K_{n, m+4}=0, m \geq 1, \\
b_{n}=\frac{\alpha_{n+1}}{\alpha_{n}}\left(K_{n+1,1}-K_{n-1,1}\right), n \geq 1, \\
c_{n}=K_{n-2,2}-K_{n, 2}-K_{n-1,1}\left(K_{n, 1}-K_{n-2,1}\right), n \geq 1, \\
a_{n}^{2}=1-K_{n-2,4}+K_{n, 4}+K_{n-1,3}\left(K_{n, 1}-K_{n-2,1}\right)-K_{n, 2}\left[K_{n, 2}-K_{n-2,2}-\right. \\
\left.-K_{n-1,1}\left(K_{n, 1}-K_{n-2,1}\right)\right]-K_{n+1,1}\left[K_{n, 3}-K_{n-2,3}-K_{n-1,2}\left(K_{n, 1}-K_{n-2,1}\right)\right]- \\
-K_{n, 1}\left[K_{n, 2}-K_{n-2,2}-K_{n-1,1}\left(K_{n, 1}-K_{n-2,1}\right)\right], n \geq 1, \\
\frac{\alpha_{n}}{\alpha_{n-2}}=a_{n-2}, n=1,2,3 .
\end{gathered}
$$

Consequently we have that if $\alpha_{n}$ and $K_{n, m}$ are defined by the equations (2.12)-(2.16) then the sequence $f_{n}(\xi)$ which is defined by Eq. (2.5) is the solution of equations (2.3). From Eq. (2.16) we immediately obtain that

$$
\alpha_{n}=\left(\prod_{p=n}^{\infty} a_{2 p-n}\right)^{-1} .
$$

Using the method of the successive approximation from the equations (2.7)-(2.11) it is not difficult to find that there exists a the unique solution $K_{n, m}$ for each $m=1,2, \ldots$ such that

$$
\left|K_{n, m}\right| \leq C \sigma\left(n+\left[\frac{m}{2}\right]\right), n, m \geq 1
$$

where,

$$
\sigma(n)=\sum_{p=n}^{\infty}\left(\left|1-a_{p}\right|+\left|b_{p}\right|+\left|c_{p}\right|\right)
$$

$C>0$ is a constant not depending on $n$ and $m$. Here [·] denotes the integer part of a reel number.

Further, from the estimation (2.18) and the continuity of the function $f_{n}(\xi)(-\infty<\xi<+\infty)$ we obtain that the function $f_{n}(\xi)$ can be analytically extended to the upper semiplane $\operatorname{Im} z>0$ by the same formula

$$
f_{n}(z)=\alpha_{n} e^{i n z}\left(1+\sum_{m=1}^{\infty} K_{n, m} e^{i m z}\right), n=1,2, \ldots ; \operatorname{Im} z>0 .
$$

Now it is easy to obtain the asymptotic formula

$$
f_{n}(z)=\alpha_{n} e^{i n z}\left(1+O\left(e^{-\tau}\right)\right), z=\xi+i \tau, \tau>0,
$$

which is uniformly on $z$. We also have

$$
f_{n}(z)=e^{i n z}(1+o(1)), n \rightarrow \infty, \quad \operatorname{Im} z \geq 0 .
$$


Finally from (2.20) we also have

$$
f_{n}(z+2 \pi)=f_{n}(z)
$$

Hence we have proved the following Theorem:

Theorem 2.1. If

$$
\sum_{n=1}^{\infty} n\left(\left|1-a_{n}\right|+\left|b_{n}\right|+\left|c_{n}\right|\right)<\infty
$$

then for each value $\lambda=2 \cos 2 \xi$ the problem (2.3)-(2.4) has the unique solution $\left(f_{n}(\xi)\right)_{n=1}^{\infty}$ which is represented as

$$
f_{n}(\xi)=\alpha_{n} e^{i n \xi}\left(1+\sum_{m=1}^{\infty} K_{n, m} e^{i m \xi}\right), n=1,2, \ldots
$$

Moreover the following conditions are satisfied:

(a) the number sequences $\alpha_{n}$ and $K_{n, m}$ satisfy equations (2.12)-(2.16) and these numbers can be defined uniquely by the sequences $\left(a_{n}\right),\left(b_{n}\right),\left(c_{n}\right)$;

(b) the estimation (2.18) is valid;

(c) $f_{n}(\xi)$ has properties $(2.20)-(2.23)$.

Note that together with $f_{n}(\xi)$ the sequences $f_{n}(-\xi), f_{n}(\pi-\xi), f_{n}(\pi+\xi)$ are also solutions of the system of equations (2.3).

\section{CONFLicts OF INTEREST}

The authors declare that there are no conflicts of interest regarding the publication of this article.

\section{REFERENCES}

[1] Guseinov, G.Sh., Determination of an infinite Jacobi matrix from scattering data, Doklady Akademii Nauk SSSR, 227(6)(1976), $1289-1292$. 1,2

[2] Guseinov, G.Sh., The inverse problem of scattering theory for a second order difference equation on the whole axis, Doklady Akademii Nauk SSSR, 17(1976), 1684-1688. 1

[3] Guseinov, G.Sh., Determination of an infinite non-self-adjoint Jacobi matrix from its generalized spectral function, Mathematical Notes, 23(2)(1978), 130-136. 1, 2

[4] Guseinov, I.M., Khanmamedov, Ag. Kh., The $t \rightarrow \infty$ asymptotic regime of the Cauchy problem solution for the Toda chain with threshold-type Initial data, Theoretical and Mathematical Physics, 119(1999), 739-749. 1

[5] Khanmamedov, Ag. Kh., Inverse scattering problem for a discrete Sturm-Liouville Operator on the entire line, Doklady Akademii Nauk, 431(1)(2010), 25-26. 1

[6] Kishakevich, Yu.L., Spectral function of Marchenko type for a difference operator of an even order, Mathematical Notes, 11(4)(1972), $266-271$. 1

[7] Marchenko, V.A., Sturm-Liouville Operators and Their Applications, Naukova Dumka, Kiev, 1977. 2, 2

[8] Zagorodnyuk, S., The direct and inverse spectral problems for $(2 N+1)$-diagonal complex transposition-antisymmetric matrices, Methods Funct. Anal. Topology, 14(2)(2008), 124-131. 1

[9] Zagorodnyuk, S.M., Direct and inverse spectral problems for $(2 N+1)$-diagonal, complex, symmetric, non-Hermitian matrices, Serdica Mathematical Journal, 30(4)(2004), 471-482. 1 